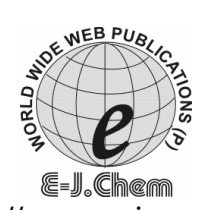

http://www.e-journals.net
ISSN: 0973-4945; CODEN ECJHAO

E-Journal of Chemistry

2008, 5(S2), 1133-1143

\title{
Synthesis, Spectral and Anthelmintic Activity Studies on Some Novel Imidazole Derivatives
}

\author{
RAJIV DAHIYA* and ANIL KUMAR \\ Department of Pharmaceutical Chemistry, \\ Rajiv Academy for Pharmacy, Mathura, Uttar Pradesh-281 001, India \\ rajivdahiya77@rediffmail.com
}

Received 2 April 2008; Revised 8 June 2008; Accepted 24 June 2008

\begin{abstract}
Present study describes the synthesis of a novel series of 3,5-diiodo-4(5-nitro-1H-2-imidazolyl)benzoyl amino acids and di/tri/tetrapeptides using diisopropylcarbodiimide/dicyclohexylcarbodiimide (DIPC/DCC) as coupling agents and $\mathrm{N}$-methylmorpholine/triethylamine (NMM/TEA) as bases. Structure elucidation of all the newly synthesized compounds was done by elemental analysis and IR, ${ }^{1} \mathrm{H}$ NMR, ${ }^{13} \mathrm{C}$ NMR and mass spectral data. Synthesized imidazolopeptides were screened for their anthelmintic activity and found to possess moderate to good bioactivity against earthworms Megascoplex konkanensis, Pontoscotex corethruses and Eudrilus eugeniea when compared to reference drugs - albendazole and mebendazole at dose level of $2 \mathrm{mg} \mathrm{mL}^{-1}$.
\end{abstract}

Keywords: 5-Nitroimidazole, 4-Amino-3,5-diiodobenzoic acid, Imidazolopeptides; Peptide coupling, Anthelmintic activity.

\section{Introduction}

Imidazole is vital heterocyclic nucleus which is well known for its wide biological profile. Imidazole derivatives are associated with a wide array of pharmacological activities including antimicrobial $^{1,2}$, anti-inflammatory and analgesic ${ }^{3,4}$, antitubercular ${ }^{5}$, cytotoxic ${ }^{6}$, antiviral ${ }^{7}$, antimuscarinic $^{8}$, FTase, gastric $\mathrm{H}^{+} / \mathrm{K}^{+}$-ATPase and MAP kinase $\mathrm{p} 38$ inhibitory activities ${ }^{9-11}$. Much work has been done on synthesis of potent polysubstituted imidazole derivatives ${ }^{12,13}$ but only few reports have been received on peptide coupling of imidazoles. Thus keeping in view the biological potency of imidazole derivatives as well as taking advantage of biodegradability and biocompatibility of amino acids/peptides and further, in continuation of our research work on synthesizing peptide derivatives of heterocyclic and other aromatic compounds ${ }^{14-26}$, a novel series of 3,5-diiodo-4-(5-nitro-1H-2-imidazolyl)benzoyl amino acids and peptides was synthesized with an anticipation to get potent agents of more therapeutic efficacy with negligible side effects. 
4-Amino-3,5-diiodobenzoic acid (1) was prepared according to literature procedure ${ }^{27}$ in good yields by iodination of $p$-aminobenzoic acid (PABA) using iodine monochloride and imidazole was nitrated using nitrating mixture by standard procedure ${ }^{28}$ to afford 5-nitroimidazole (2). 3,5-Diiodo-4-(5-nitro-1H-2-imidazolyl)benzoic acid (3) was synthesized by diazotization of (1) followed by subsequent interaction with (2) in presence of aqueous cupric chloride $\left(\mathrm{CuCl}_{2}\right)$. Dipeptides Boc-Ala-Pro-OMe, Boc-Phe-Val-OMe and Boc-TryHis-OMe were prepared from the corresponding Boc-amino acids viz. Boc-Ala, Boc-Phe and Boc-Try, and amino acid methyl ester hydrochlorides such as Pro-OMe.HCl, Val$\mathrm{OMe} . \mathrm{HCl}$ and His-OMe.HCl using DCC and TEA in dichloromethane ${ }^{29}$. Similarly, tripeptides Boc-Pro-Pro-Pro-OMe and tetrapeptide Boc-Ala-His-Gly-Try-OMe was prepared by coupling Boc-dipeptides with corresponding amino acid methyl ester hydrochlorides /dipeptide methyl ester. Prior to coupling, all Boc-di/tri/tetrapeptide methyl esters were deprotected at amino terminals using trifluoroacetic acid (TFA).

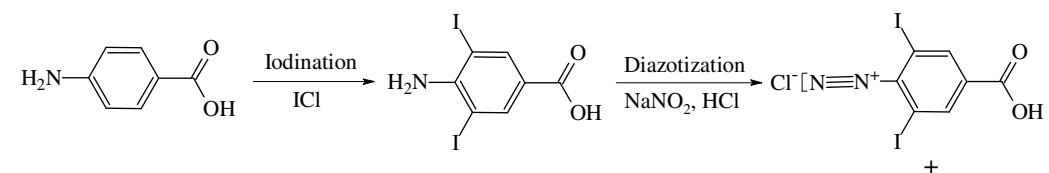

(1)

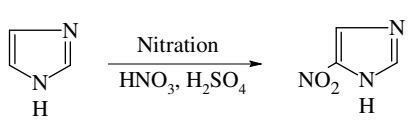

(2)

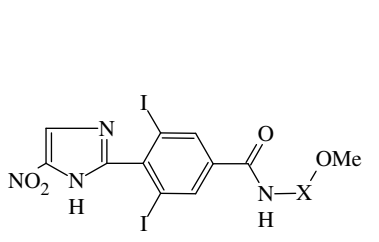

(4-11)

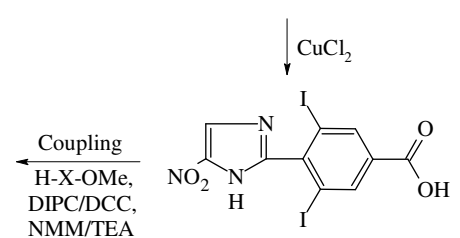

(3)

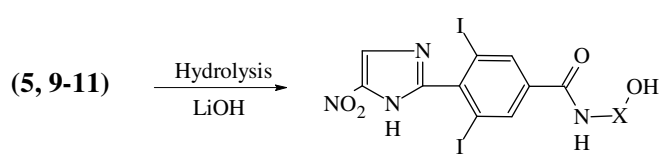

(12-15)

$\mathrm{X}=$ nitro(Arg) (4), Tyr (5), Try (6), Ala-Pro (7), Phe-Val (8), Try-His (9), Pro-Pro-Pro (10), Ala-His-Gly-Try (11), Tyr (12), Try-His (13), Pro-Pro-Pro (14), Ala-His-Gly-Try (15).

Scheme 1. Synthesis of 3,5-diiodo-4-(5-nitro-1H-2-imidazolyl)benzoyl amino acids/peptides. 3,5-Diiodo-4-(5-nitro-1H-2-imidazolyl)benzoic acid (3) was coupled with various L-amino acid methyl ester hydrochlorides/peptide methyl esters using DIPC/DCC and NMM/TEA to get newly synthesized 3,5-diiodo-4-(5-nitro-1H-2-imidazolyl)benzoyl amino acid methyl esters (4-6), dipeptide methyl esters (7-9), tripeptide methyl ester (10) and tetrapeptide methyl ester (11). Finally, selected ester derivatives (4, 9-11) were hydrolyzed with lithium hydroxide ( $\mathrm{LiOH})$ to get the corresponding acid derivatives (12-15) (Scheme 1). Structures of all the newly synthesized compounds were confirmed by IR, ${ }^{1} \mathrm{H}$ NMR, ${ }^{13} \mathrm{C}$ NMR and mass spectral data. Elemental analysis of the novel compounds was performed for carbon, hydrogen and nitrogen content. 


\section{Experimental}

Melting points were determined by open capillary method and are uncorrected. Amino acids, di-tert-butylpyrocarbonate $\left(\mathrm{Boc}_{2} \mathrm{O}\right)$, DIPC, DCC, TFA, NMM and TEA were procured from SpectroChem Ltd, Mumbai, India. IR spectra were recorded on Shimadzu 8700 fourier transform infrared spectrophotometer using a thin film supported on $\mathrm{KBr}$ pellets for solids and $\mathrm{CDCl}_{3}$ as solvent for semisolids. ${ }^{1} \mathrm{H}$ NMR and ${ }^{13} \mathrm{C}$ NMR spectra were recorded on Bruker AC NMR spectrometer $(300 \mathrm{MHz})$ using $\mathrm{CDCl}_{3}$ as solvent and TMS as internal standard. Mass spectra were recorded on Jeol JMS DX 303 Mass spectrometer operating at $70 \mathrm{eV}$. Elemental analysis of all compounds was performed on Elementar vario EL III. Purity of all the compounds was checked by TLC on precoated silica gel G plates. Optical rotation of synthesized peptide derivatives was measured on automatic polarimeter (Optics Tech, Ghaziabad, India) in a $2 \mathrm{dm}$ tube at $25^{\circ} \mathrm{C}$ using sodium D-light and methanol as solvent.

\section{Preparation of peptides}

Amino acid methyl ester hydrochloride/dipeptide methyl ester $(0.01 \mathrm{~mol})$ was dissolved in $\mathrm{CH}_{2} \mathrm{Cl}_{2}(20 \mathrm{~mL})$. To this, TEA $(0.021 \mathrm{~mol})$ was added at $0^{\circ} \mathrm{C}$ and the reaction mixture was stirred for $15 \mathrm{~min}$. Boc-amino acid/dipeptide $(0.01 \mathrm{~mol})$ in $\mathrm{CH}_{2} \mathrm{Cl}_{2}(20 \mathrm{~mL})$ and DCC $(0.01$ mol) were added with stirring. After $24 \mathrm{~h}$, the reaction mixture was filtered and the residue was washed with $\mathrm{CHCl}_{3}(30 \mathrm{~mL})$ and added to the filtrate. The filtrate was washed with $5 \%$ $\mathrm{NaHCO}_{3}$ and saturated $\mathrm{NaCl}$ solutions. The organic layer was dried over anhydrous $\mathrm{Na}_{2} \mathrm{SO}_{4}$, filtered and evaporated in vacuum. The crude product was recrystallized from a mixture of chloroform and $n$-hexane followed by cooling at $0^{\circ} \mathrm{C}$. Resulting Boc-di/tri/tetrapeptide methyl ester $(0.01 \mathrm{~mol})$ was dissolved in $\mathrm{CHCl}_{3}(15 \mathrm{~mL})$ and treated with trifluoroacetic acid $(0.020$ mol). The mixture was stirred at room temperature for $1 \mathrm{~h}$ and washed with saturated $\mathrm{NaHCO}_{3}$ solution. The resulting organic layer was dried over anhydrous $\mathrm{Na}_{2} \mathrm{SO}_{4}$ and concentrated under reduced pressure. Finally, crude product was purified by recrystallization with $\mathrm{CHCl}_{3}$ and petroleum ether (B.p. $40-60^{\circ} \mathrm{C}$ ) to get the deprotected di/tri/tetrapeptide methyl esters.

Preparation of 3,5-diiodo-4-(5-nitro-1H-2-imidazolyl)benzoic acid (3)

A mixture of 4-amino-3,5-diiodobenzoic acid (1) (38.9 g, $0.1 \mathrm{~mol}$ ), dilute hydrochloric acid $(15 \%, 60 \mathrm{~mL})$ and water $(90 \mathrm{~mL})$ was heated to get a clear solution. After cooling, the solution was diazotized by addition of sodium nitrite solution $(30 \%, 24 \mathrm{~mL})$. To the filtrate of diazotized salt solution, 5-nitroimidazole (2) (11.3 gm, $0.01 \mathrm{~mol})$ and aqueous cupric chloride solution $(2.5 \mathrm{~g}$ in $10 \mathrm{~mL}$ of water) were added with stirring followed by slow addition of water $(50 \mathrm{~mL})$. Stirring was continued for $4 \mathrm{~h}$ and kept overnight in the refrigerator. The separated solid was collected by filtration and washed with cold water. The crude product was recrystallized from acetone to get the title compound. White crystals; Yield 74\%; m.p. $123-125{ }^{\circ} \mathrm{C} ; \mathrm{R}_{f}-0.63\left(\mathrm{CHCl}_{3}: \mathrm{AcOH}: \mathrm{H}_{2} \mathrm{O} / 3: 2: 5\right)$; IR $(\mathrm{KBr})\left(\mathrm{cm}^{-1}\right): 3489$ (N-H stretching (str), ring), 3302-2497 (O-H str, COOH), 3079, $3056(\mathrm{Ar}-\mathrm{H}$ str), $1698(\mathrm{C}=\mathrm{O}$ str, $\mathrm{COOH}), 1589,1574,1429,1417$ (skeletal bands), 1545, $1348\left(\mathrm{NO}_{2}\right.$ str), $1405(\mathrm{O}-\mathrm{H}$ deformation (def), COOH), 875, 833, 824, 696 (C-H def, out-of-plane (oop)), 590 (C-I str);

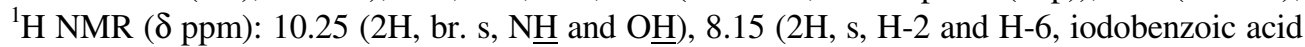
(iba)), $7.85\left(1 \mathrm{H}, \mathrm{s}, \mathrm{H}-\delta\right.$, imidazole (imz)); ${ }^{13} \mathrm{C}$ NMR $(\delta \mathrm{ppm}): 170.4(\mathrm{C}=\mathrm{O}, \underline{\mathrm{COOH}}), 152.8(\mathrm{C}-\varepsilon$, imz), 148.7 (C-4, iba), 143.1 (2C, C-2 and C-6, iba), 137.3 (C- $\delta, i m z), 132.6$ (C-1, iba), 109.4 (2C, C-3 and C-5, iba); Anal. calcd. for $\mathrm{C}_{10} \mathrm{H}_{5} \mathrm{I}_{2} \mathrm{~N}_{3} \mathrm{O}_{4}$ (484): C, 24.77; H, 1.04; N, 8.66. Found: C, 24.75; H, 1.06; N, 8.65\%. 
Preparation of 3,5-diiodo-4-(5-nitro-1H-2-imidazolyl)benzoyl amino acid/peptide methyl esters (4-11)

To a mixture of amino acid methyl ester hydrochloride/di/tri/tetrapeptide methyl ester $(0.01$ mol) in DMF $(50 \mathrm{~mL}), \mathrm{NMM} / \mathrm{TEA}(2.3 \mathrm{~mL} / 2.8 \mathrm{~mL})$ was added at $0{ }^{\circ} \mathrm{C}$ with stirring. Compound (3) $(4.85 \mathrm{gm}, 0.01 \mathrm{~mol})$ in DMF $(50 \mathrm{~mL})$ and DIPC/DCC $(1.26 \mathrm{gm} / 2.1 \mathrm{gm})$ were added to the above mixture and stirring was done for $24 \mathrm{~h}$. The reaction mixture was filtered and diluted with equal proportion of water. The aqueous layer was washed with ether $(3 \times 50$ $\mathrm{mL}$ ) and organic layer was dried over anhydrous $\mathrm{Na}_{2} \mathrm{SO}_{4}$, filtered and evaporated. The product obtained was dissolved in chloroform, washed with $10 \% \mathrm{HCl}$, saturated $\mathrm{NaHCO}_{3}$ solution and water $(25 \mathrm{~mL}$ each) followed by evaporation in vacuum. Crude product was crystallized from a mixture of ethyl acetate and petroleum ether (B.p. 40-60 ${ }^{\circ} \mathrm{C}$ ).

\section{3,5-Diiodo-4-(5-nitro-1H-2-imidazolyl)benzoyl nitro(arginine) methyl ester (4)}

Semisolid mass; Yield 75\% (DIPC/NMM), 69\% (DCC/TEA); $[\alpha]_{\mathrm{D}}-59.8^{\circ}(\mathrm{c} 0.3, \mathrm{MeOH}) ; \mathrm{R}_{f}$ $0.83\left(\mathrm{CHCl}_{3}: \mathrm{MeOH} / 9: 1\right) ; \mathrm{IR}\left(\mathrm{CDCl}_{3}\right)\left(\mathrm{cm}^{-1}\right): 3485(\mathrm{~N}-\mathrm{H}$ str, ring), 3340, 3327 (N-H str, amine), 3126 (N-H str, amide), 3075, 3052 (Ar-H str), 2928-2922, 2855, 2849 (C-H str, $\mathrm{CH}_{2}$ ), 1747 (C=O str, ester), 1638 ( $\mathrm{C}=\mathrm{O}$ str, amide), 1587, 1576, 1428, 1417 (skeletal bands), 1547, 1544, $1349\left(\mathrm{NO}_{2}\right.$ str), 1532 ( $\mathrm{N}-\mathrm{H}$ def, amide), 1468, 1459 (C-H def (scissoring), $\left.\mathrm{CH}_{2}\right), 1269$ (C-O str, ester), 1154 (C-N str, amine), 876, 831, 822, 695 (C-H def, oop), 588 (C-I str); ${ }^{1} \mathrm{H}$ NMR $(\delta$ ppm):

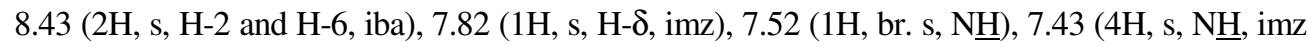
and $\operatorname{Arg}$ ), 4.38-4.33 (1H, m, H- $\alpha$, Arg), 3.88-4.83 (2H, m, H- $\left.\delta, \operatorname{Arg}), 3.65\left(3 \mathrm{H}, \mathrm{s}, \mathrm{OC}_{3}\right)_{3}\right), 1.98-$ 1.93 (2H, q, H- $\beta, \operatorname{Arg}), 1.67-1.62$ (2H, m, H- $\gamma, \operatorname{Arg}) ;{ }^{13} \mathrm{C}$ NMR $(\delta$ ppm): $171.9(\mathrm{C}=\mathrm{O}, \mathrm{Arg}), 170.2$ $(\mathrm{C}=\mathrm{O}, \mathrm{Ar} \underline{\mathrm{CO}}), 162.5(\underline{\mathrm{C}}=\mathrm{NH}), 154.2(\mathrm{C}-\beta, \mathrm{imz}), 151.9(\mathrm{C}-\varepsilon, \mathrm{imz}), 146.0(\mathrm{C}-4, \mathrm{iba}), 144.6(2 \mathrm{C}, \mathrm{C}-2$ and C-6, iba), 138.2 (C-1, iba), 137.3 (C- $\delta, \mathrm{imz}), 108.2$ (2C, C-3 and C-5, iba), 54.3 (C- $\alpha, \mathrm{Arg}$ ), $51.1\left(\mathrm{OCH}_{3}\right), 40.2(\mathrm{C}-\delta, \mathrm{Arg}), 27.8(\mathrm{C}-\beta, \mathrm{Arg}), 24.2(\mathrm{C}-\gamma, \mathrm{Arg}) ;$ Anal. calcd. for $\mathrm{C}_{17} \mathrm{H}_{18} \mathrm{I}_{2} \mathrm{~N}_{8} \mathrm{O}_{7}$ (700): C, 29.16; H, 2.59; N, 16.00. Found: C, 29.15; H, 2.62; N, $15.97 \%$.

\section{3,5-Diiodo-4-(5-nitro-1H-2-imidazolyl)benzoyl tyrosine methyl ester (5)}

White crystals; Yield 79\% (DIPC/NMM), 72\% (DCC/TEA); m.p. 152-153 ${ }^{\circ} \mathrm{C} ;[\alpha]_{\mathrm{D}}-17.3^{\circ}$ (c 0.3, MeOH); $\mathrm{R}_{f}-0.55\left(\mathrm{CHCl}_{3}: \mathrm{MeOH} / 9: 1\right)$; IR $(\mathrm{KBr})\left(\mathrm{cm}^{-1}\right): 3488(\mathrm{~N}-\mathrm{H}$ str, ring), 3359 (O-H str, Ar-OH), 3122 (N-H str, amide), 3077, 3055-3052 (Ar-H str), 2925, 2852 (C-H str, $\left.\mathrm{CH}_{2}\right), 1749(\mathrm{C}=\mathrm{O}$ str, ester), $1642(\mathrm{C}=\mathrm{O}$ str, amide), 1589-1578, 1425-1418 (skeletal bands), 1541, 1346 ( $\mathrm{NO}_{2}$ str), 1535 ( $\mathrm{N}-\mathrm{H}$ def, amide), 1272 (C-O str, ester), 1224 (C-O str, phenolic), 872, 828-822, 763, 697 (C-H def, oop), 586 (C-I str); ${ }^{1} \mathrm{H}$ NMR ( $\delta$ ppm): 8.42

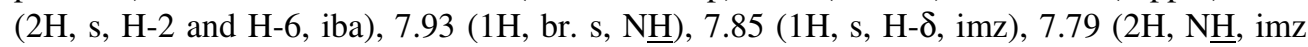
and $\mathrm{OH}, \mathrm{Tyr}), 6.92(2 \mathrm{H}, \mathrm{dd}, J=8.55,5.25 \mathrm{~Hz}, \mathrm{H}-\mathrm{o}, \mathrm{Tyr}), 6.80(2 \mathrm{H}, \mathrm{dd}, J=8.6,4.9 \mathrm{~Hz}, \mathrm{H}-\mathrm{m}$, Tyr), 4.68-4.62 (1H, m, H- $\alpha$, Tyr), $3.53\left(3 \mathrm{H}, \mathrm{s}, \mathrm{OCH}_{3}\right), 2.98(2 \mathrm{H}, \mathrm{d}, J=5.45 \mathrm{~Hz}, \mathrm{H}-\beta, \mathrm{Tyr})$; ${ }^{13} \mathrm{C}$ NMR $(\delta \mathrm{ppm}): 170.6(\mathrm{C}=\mathrm{O}, \mathrm{Tyr}), 168.8(\mathrm{C}=\mathrm{O}, \mathrm{ArCO}), 154.5(\mathrm{C}-\varepsilon, \mathrm{imz}), 153.9(\mathrm{C}-\mathrm{p}$, Tyr), 152.7 (C- $\beta$, imz), 145.6 (C-4, iba), 142.0 (2C, C-2 and C-6, iba), 138.8 (C-1, iba), 138.2 (2C, C-m, Tyr), 137.0 (C- $\delta$, imz), 130.2 (2C, C-o, Tyr), 127 (C- $\gamma$, Tyr), 107.6 (2C, C3 and $\mathrm{C}-5$, iba), $57.5(\mathrm{C}-\alpha, \mathrm{Tyr}), 54.4\left(\mathrm{OCH}_{3}\right), 38.4(\mathrm{C}-\beta, \mathrm{Tyr})$; Anal. calcd. for $\mathrm{C}_{20} \mathrm{H}_{16} \mathrm{I}_{2} \mathrm{~N}_{4} \mathrm{O}_{6}$ (662): C, 36.28; H, 2.44; N, 8.46. Found: C, 36.25; H, 2.46; N, 8.47\%.

\section{3,5-Diiodo-4-(5-nitro-1H-2-imidazolyl)benzoyl tryptophan methyl ester (6)}

White solid; Yield $83 \%$ (DIPC/NMM), $75 \%$ (DCC/TEA); m.p. $184-186{ }^{\circ} \mathrm{C}$; $[\alpha]_{\mathrm{D}}+1.9^{\circ}$ (c 0.3, MeOH); $\mathrm{R}_{f}-0.69\left(\mathrm{CHCl}_{3}: \mathrm{MeOH} / 9: 1\right)$; IR (KBr) $\left(\mathrm{cm}^{-1}\right): 3489,3485(\mathrm{~N}-\mathrm{H}$ str, rings), 3125 (N-H str, amide), 3079-3074, 3056 (Ar-H str), 2926, 2853 (C-H str, $\left.\mathrm{CH}_{2}\right), 1752$ 
(C=O str, ester), 1644 ( $\mathrm{C}=\mathrm{O}$ str, amide), 1588-1576, 1429-1415 (skeletal bands), 1541, $1349\left(\mathrm{NO}_{2}\right.$ str), 1536 (N-H def, amide), 1268 (C-O str, ester), 879-873, 832-823, 692 (C-H def, oop), 586 (C-I str); ${ }^{1} \mathrm{H}$ NMR ( $\delta$ ppm): $9.32(2 \mathrm{H}, \mathrm{NH}, \mathrm{imz}$ and indole), $8.42(2 \mathrm{H}, \mathrm{s}, \mathrm{H}-2$ and $\mathrm{H}-6$, iba), $7.93(1 \mathrm{H}$, br. s, $\mathrm{N} \underline{\mathrm{H}}), 7.85(1 \mathrm{H}, \mathrm{s}, \mathrm{H}-\delta, \mathrm{imz}), 7.52(1 \mathrm{H}, \mathrm{d}, J=7.8 \mathrm{~Hz}, \mathrm{H}-2$, Try), 7.14-7.05 (4H, m, H-4-7, Try), 4.94-4.89 (1H, m, H- $\alpha$, Try), 3.55 (3H, s, OC$\left.\underline{H}_{3}\right), 3.21$ $\left(2 \mathrm{H}, \mathrm{d}, J=5.7 \mathrm{~Hz}, \mathrm{H}-\beta\right.$, Try); ${ }^{13} \mathrm{C}$ NMR $(\delta \mathrm{ppm}): 175.6(\mathrm{C}=\mathrm{O}$, Try $), 169.7$ (C=O, ArCO), 155.3 (C- $\beta$, imz), 153.6 (C- $\varepsilon$, imz), 148.2 (C-4, iba), 143.1 (2C, C-2 and C-6, iba), 137.6 (C2', Try), 135.1 (C- $\delta$, imz), 134.6 (C-1, iba), 128.1 (C-3', Try), 122.8 (C-2, Try), 121.9 (C-6, Try), 119.5 (C-5, Try), 118.2 (C-4, Try), 115.4 (C-3, Try), 111.6 (C-7, Try), 106.8 (2C, C-3 and C-5, iba), 56.7 (C- $\alpha$, Try), $51.4\left(\mathrm{OCH}_{3}\right), 22.4$ (C- $\beta$, Try); Anal. calcd. for $\mathrm{C}_{22} \mathrm{H}_{17} \mathrm{I}_{2} \mathrm{~N}_{5} \mathrm{O}_{5}$ (685): C, 38.56; H, 2.50; N, 10.22. Found: C, 38.55; H, 2.53; N, 10.25\%.

\section{3,5-Diiodo-4-(5-nitro-1H-2-imidazolyl)benzoyl alanyl-proline methyl ester (7)}

Semisolid mass; Yield 74\% (DIPC/NMM), 68\% (DCC/TEA); $[\alpha]_{\mathrm{D}}-91.7^{\circ}$ (c 0.3, MeOH); $\mathrm{R}_{f}-0.49\left(\mathrm{CHCl}_{3}: \mathrm{MeOH} / 9: 1\right)$; IR $\left(\mathrm{CDCl}_{3}\right)\left(\mathrm{cm}^{-1}\right): 3487(\mathrm{~N}-\mathrm{H}$ str, ring), $3123(\mathrm{~N}-\mathrm{H}$ str, amide), 3078, 3055 ( $\mathrm{Ar}-\mathrm{H}$ str), 2994-2988 (C-H str, $\mathrm{CH}_{2}$, Pro), 2962, 2874 (C-H str, $\mathrm{CH}_{3}$ ), 1753 ( $\mathrm{C}=\mathrm{O}$ str, ester), 1658, 1639 ( $\mathrm{C}=\mathrm{O}$ str, amide), 1589, 1575, 1425, 1419 (skeletal bands), 1549, 1544, 1345 ( $\mathrm{NO}_{2}$ str), 1536 ( $\mathrm{N}-\mathrm{H}$ def, amide), 1267 (C-O str, ester), 878, 833, 821, 696 (C-H def, oop), 586 (C-I str); ${ }^{1} \mathrm{H}$ NMR ( $\delta$ ppm): 9.69 (1H, br. s, N태, imz), 8.50 (2H, s,

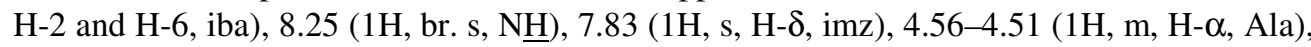
$3.89\left(1 \mathrm{H}, \mathrm{t}, J=6.9 \mathrm{~Hz}, \mathrm{H}-\alpha\right.$, Pro), $3.62\left(3 \mathrm{H}, \mathrm{s}, \mathrm{OC}_{\underline{3}}\right), 3.39(2 \mathrm{H}, \mathrm{t}, J=7.15 \mathrm{~Hz}, \mathrm{H}-\delta$, Pro), 2.05-1.97 (4H, m, H- $\gamma$ and $\mathrm{H}-\delta$, Pro), $1.45(3 \mathrm{H}, \mathrm{d}, J=5.9 \mathrm{~Hz}, \mathrm{H}-\beta, \mathrm{Ala}) ;{ }^{13} \mathrm{C}$ NMR $(\delta \mathrm{ppm})$ : $174.3(\mathrm{C}=\mathrm{O}, \operatorname{Pro}), 169.6(\mathrm{C}=\mathrm{O}, \operatorname{Ar} \underline{C} \mathrm{O}), 165.1(\mathrm{C}=\mathrm{O}, \mathrm{Ala}), 155.0(\mathrm{C}-\beta, \mathrm{imz}), 153.2(\mathrm{C}-\varepsilon$, imz), 147.7 (C-4, iba), 144.0 (2C, C-2 and C-6, iba), 140.4 (C-1, iba), 136.6 (C- $\delta$, imz), 108.0 (2C, C-3 and C-5, iba), 59.3 (C- $\alpha$, Pro), $54.0\left(\mathrm{OCH}_{3}\right), 53.6$ (C- $\alpha$, Ala), 45.2 (C- $\delta$, Pro), 29.1 (C- $\beta$, Pro), 25.7 (C- $\gamma$, Pro), 18.5 (C- $\beta$, Ala); Anal. calcd. for $\mathrm{C}_{19} \mathrm{H}_{19} \mathrm{I}_{2} \mathrm{~N}_{5} \mathrm{O}_{6}$ (667): C, 34.20; H, 2.87; N, 10.50. Found: C, 34.19; H, 2.85; N, $10.53 \%$.

\section{3,5-Diiodo-4-(5-nitro-1H-2-imidazolyl)benzoyl phenylalanyl-valine methyl ester (8)}

Off-white solid; Yield 84\% (DIPC/NMM), 77\% (DCC/TEA); m.p. 98-99 ${ }^{\circ} \mathrm{C} ;[\alpha]_{\mathrm{D}}-112.3^{\circ}$ (c 0.3, MeOH); $\mathrm{R}_{f}-0.72\left(\mathrm{CHCl}_{3}: \mathrm{MeOH} / 9: 1\right) ; \mathrm{IR}(\mathrm{KBr})\left(\mathrm{cm}^{-1}\right): 3488(\mathrm{~N}-\mathrm{H}$ str, ring), 3128 , 3122 (N-H str, amide), 3079, 3055-3051 (Ar-H str), 2966 (C-H str, $\mathrm{CH}_{3}$ ), 2929, 2853 (C-H str, $\mathrm{CH}_{2}$ ), 1748 (C=O str, ester), 1644, 1640 (C=O str, amide), 1589-1577, 1429-1418 (skeletal bands), 1544, 1341 ( $\mathrm{NO}_{2}$ str), 1536, 1529 (N-H def, amide), 1385, $1362(\mathrm{C}-\mathrm{H}$ bend, propyl-i), 1273 (C-O str, ester), 872, 839, 819, 715, 698, 692 (C-H def, oop), 588

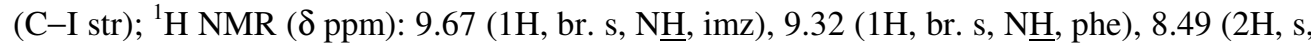
$\mathrm{H}-2$ and H-6, iba), 7.85 (1H, s, H- $\delta$, imz), 7.22-7.17 (2H, m, H-m, Phe), 6.98 (1H, t, $J=6.2$

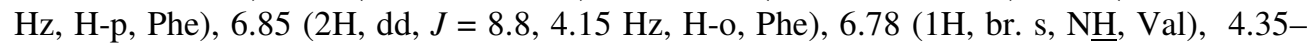
4.31 (1H, m, H- $\alpha$, Phe), $3.72(1 \mathrm{H}, \mathrm{dd}, J=5.4,3.75 \mathrm{~Hz}, \mathrm{H}-\alpha, \mathrm{Val}), 3.66\left(3 \mathrm{H}, \mathrm{s}, \mathrm{OC}_{3}\right), 2.82$ $(2 \mathrm{H}, \mathrm{t}, J=5.65 \mathrm{~Hz}, \mathrm{H}-\beta, \mathrm{Phe}), 2.17-2.11(1 \mathrm{H}, \mathrm{m}, \mathrm{H}-\beta, \mathrm{Val}), 0.82(6 \mathrm{H}, \mathrm{d}, J=4.5 \mathrm{~Hz}, \mathrm{H}-\gamma$, Val $) ;{ }^{13} \mathrm{C}$ NMR $(\delta$ ppm): $175.2(\mathrm{C}=\mathrm{O}, \mathrm{Val}), 171.5(\mathrm{C}=\mathrm{O}, \mathrm{Phe}), 169.0(\mathrm{C}=\mathrm{O}$, ArCO $), 155.7$ (C- $\beta$, imz), 153.9 (C- $\varepsilon, \mathrm{imz}), 147.7$ (C-4, iba), 143.3 (2C, C-2 and C-6, iba), 142.0 (C- $\gamma$, Phe), 140.2 (C-1, iba), 136.8 (C- $\delta$, imz), 130.2 (2C, C-o, Phe), 128.9 (2C, C-m, Phe), 127.2 (C-p, Phe), 109.0 (2C, C-3 and C-5, iba), 60.3 (C- $\alpha$, Val $), 57.0\left(\mathrm{OCH}_{3}\right), 55.6(\mathrm{C}-\alpha, \mathrm{Phe}), 40.8(\mathrm{C}-$ $\beta$, Phe), 31.9 (C- $\beta$, Val), 21.2 (2C, C- $\gamma$, Val); Anal. calcd. for $\mathrm{C}_{25} \mathrm{H}_{25} \mathrm{I}_{2} \mathrm{~N}_{5} \mathrm{O}_{6}(745)$ : C, 40.29; H, 3.38; N, 9.40. Found: C, 40.26; H, 3.40; N, 9.44\%. 
3,5-Diiodo-4-(5-nitro-1H-2-imidazolyl)benzoyl tryptophanyl-histidine methyl ester (9) White crystals; Yield 76\% (DIPC/NMM), 66\% (DCC/TEA); m.p. 201-202 ${ }^{\circ} \mathrm{C} ;[\alpha]_{\mathrm{D}}+51.5^{\circ}(\mathrm{c} 0.3$, $\mathrm{MeOH}) ; \mathrm{R}_{f}-0.78\left(\mathrm{CHCl}_{3}: \mathrm{MeOH} / 9: 1\right) ; \mathrm{IR}(\mathrm{KBr})\left(\mathrm{cm}^{-1}\right): 3489-3483(\mathrm{~N}-\mathrm{H}$ str, ring), 3124, 3319 (N-H str, amide), 3079-3072, 3054 (Ar-H str), 2929-2925, 2853, 2847 (C-H str, $\mathrm{CH}_{2}$ ), 1751 ( $\mathrm{C}=\mathrm{O}$ str, ester), 1645, 1642 ( $\mathrm{C}=\mathrm{O}$ str, amide), 1586-1572, 1428-1415 (skeletal bands), 1542, 1344 ( $\mathrm{NO}_{2}$ str), 1535-1531 ( $\mathrm{N}-\mathrm{H}$ def, amide), 1464 (C-H bend, $\left.\mathrm{CH}_{2}\right), 1273$ (C-O str, ester), 878-875, 832-826, 693 (C-H def, oop), 585 (C-I str); ${ }^{1} \mathrm{H}$ NMR ( $\delta$ ppm): 9.38 (1H, br. s, N $\underline{H}$, Try), 9.25 (3H, br. s, NH, imz, His and Try), $8.52(2 \mathrm{H}, \mathrm{s}, \mathrm{H}-2$ and H-6, iba), $7.85(1 \mathrm{H}, \mathrm{s}, \mathrm{H}-\delta$, imz), $7.75(1 \mathrm{H}, \mathrm{d}, J=7.95 \mathrm{~Hz}, \mathrm{H}-2$, His), $7.58(1 \mathrm{H}, \mathrm{s}, \mathrm{H}-4, \mathrm{His}), 7.25(1 \mathrm{H}, \mathrm{d}, J=7.75 \mathrm{~Hz}, \mathrm{H}-2$, Try), 7.22 (1H, d, J = 7.5 Hz, H-4, Try), 7.15-7.09 (3H, m, H-5-7, Try), 6.92 (1H, br. s, N $\underline{\text { }}$, His), 4.89-4.85 (1H, m, H- $\alpha$, Try), 4.15-4.10 (1H, m, H- $\alpha$, His), 3.57 (3H, s, OCH $\left.\underline{H}_{3}\right), 3.20(2 \mathrm{H}, \mathrm{d}$, $J=5.75 \mathrm{~Hz}, \mathrm{H}-\beta$, Try $), 3.05(2 \mathrm{H}, \mathrm{d}, J=5.9 \mathrm{~Hz}, \mathrm{H}-\beta, \mathrm{His}) ;{ }^{13} \mathrm{C}$ NMR $(\delta \mathrm{ppm}): 177.2(\mathrm{C}=\mathrm{O}, \mathrm{His})$, 172.6 (C=O, Try), 170.1 (C=O, ArCO), 155.0 (C- $\beta$, imz), 153.6 (C- $\varepsilon$, imz), 149.3 (C-2, His), 147.1 (C-4, iba), 143.6 (2C, C-2 and C-6, iba), 140.2 (C-1, iba), 136.4 (C-2', Try), 137.0 (C- $\delta$, imz), 135.8 (C-4, His), 129.2 (C-3', Try), 124.6 (C-2, Try), 124.0, 123.4 (2C, C-5 and C-6, Try), 119.6 (C-4, Try), 115.3 (C-5, His), 111.8 (C-3, Try), 111.2 (C-7, Try), 106.9 (2C, C-3 and C-5, iba), 65.3 (C- $\alpha$, Try), 57.1 (C- $\alpha$, His), $51.4\left(\mathrm{OCH}_{3}\right), 31.8$ (C- $\beta$, His), 24.2 (C- $\beta$, Try); Anal. calcd. for $\mathrm{C}_{28} \mathrm{H}_{24} \mathrm{I}_{2} \mathrm{~N}_{8} \mathrm{O}_{6}$ (822): C, 40.90; H, 2.94; N, 13.63. Found: C, 40.89; H, 2.95; N, $13.65 \%$.

3,5-Diiodo-4-(5-nitro-1H-2-imidazolyl)benzoyl prolyl-prolyl-proline methyl ester (10)

Semisolid mass; Yield 81\% (DIPC/NMM), 76\% (DCC/TEA); $[\alpha]_{\mathrm{D}}+24.8^{\circ}(\mathrm{c} 0.3, \mathrm{MeOH}) ; \mathrm{R}_{f}$ $0.51\left(\mathrm{CHCl}_{3}: \mathrm{MeOH} / 9: 1\right)$; IR $\left(\mathrm{CDCl}_{3}\right)\left(\mathrm{cm}^{-1}\right): 3489(\mathrm{~N}-\mathrm{H}$ str, ring), 3076, 3050 ( $\mathrm{Ar}-\mathrm{H} \mathrm{str})$, 2998-2989 (C-H str, $\mathrm{CH}_{2}$, pro), 1749 (C=O str, ester), 1662-1657 (C=O str, amide), 1589, 1571, 1429, 1416 (skeletal bands), 1545, 1349 ( $\mathrm{NO}_{2}$ str), 1272 (C-O str, ester), 879, 830, 825, 697 (C-H def, oop), 586 (C-I str); ${ }^{13} \mathrm{C}$ NMR $(\delta$ ppm): 174.1 (C=O, Pro-3), 172.5 (C=O, Pro-1), 169.2 (C=O, ArCO $), 168.1(\mathrm{C}=\mathrm{O}$, Pro-2), 155.1 (C- $\beta$, imz), 154.0 (C- $\varepsilon, \mathrm{imz}), 147.7$ (C-4, iba), 144.0 (2C, C-2 and C-6, iba), 139.9 (C-1, iba), 136.2 (C- $\delta$, imz), 107.6 (2C, C-3 and C-5, iba), 64.3, 62.6, 59.1 (3C, C- $\alpha$, Pro-1-3), $54.4\left(\mathrm{OCH}_{3}\right), 47.8,46.2,44.5$ (3C, C- $\delta$, Pro-1-3), 31.6, 29.2, 28.7 (3C, C- $\beta$, Pro-1-3), 27.3, 25.1, 24.5 (3C, C- $\gamma$, Pro-1-3); MASS (m/z \%RI): 792(2.3), 791(3.5), 790( $\left.\mathrm{M}^{+}, 4.8\right), 775(19.5), 759(32.8), 731(11.3), 662(58.2), 634(19.5), 565(100)$, 537(23.8), 467(67.4), 439(29.5), 328(11.4), 203(10.7), 126(6.8), 113(9.5), 112 (14.1), 86(17.4), 85(7.9), 59(18.5), 46(8.4), 42(19.7), 31(12.3), 15(2.4); Anal. calcd. for $\mathrm{C}_{26} \mathrm{H}_{28} \mathrm{I}_{2} \mathrm{~N}_{6} \mathrm{O}_{7}$ (790): $\mathrm{C}$, 39.51; H, 3.57; N, 10.63. Found: C, 39.48; H, 3.60; N, 10.65\%.

\section{3,5-Diiodo-4-(5-nitro-1H-2-imidazolyl)benzoyl alanyl-histidinyl-glycyl-tryptophan methyl ester (11)}

Semisolid mass; Yield 84\% (DIPC/NMM), 78\% (DCC/TEA); $[\alpha]_{\mathrm{D}}-62.4^{\circ}$ (c 0.3, MeOH); $\mathrm{R}_{f}$ $0.86\left(\mathrm{CHCl}_{3}: \mathrm{MeOH} / 9: 1\right)$; IR $\left(\mathrm{CDCl}_{3}\right)\left(\mathrm{cm}^{-1}\right): 3489-3484$ (N-H str, rings), 3128, 3123, 3119 (N-H str, amide), 3079-3074, 3054 (Ar-H str), 2965, 2872 (C-H str, $\mathrm{CH}_{3}$ ), 2929-2925, 2852, 2849 (C-H str, $\mathrm{CH}_{2}$ ), 1750 (C=O str, ester), 1644-1639 (C=O str, amide), 1589-1577, 1429-1418 (skeletal bands), 1541, 1345 ( $\mathrm{NO}_{2}$ str), 1537-1532 (N-H def, amide), $1462\left(\mathrm{C}-\mathrm{H}\right.$ bend, $\left.\mathrm{CH}_{2}\right)$, 1270 (C-O str, ester), 879-874, 830-825, 696 (C-H def, oop), 589 (C-I str); ${ }^{1} \mathrm{H}$ NMR $(\delta \mathrm{ppm})$ :

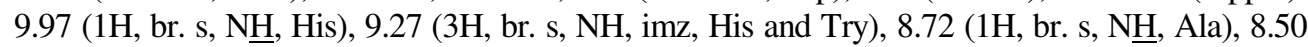
(2H, s, H-2 and H-6, iba), 8.27 (1H, br. s, N트, Gly), 7.84 (1H, s, H- $\delta$, imz), 7.64 (1H, s, H-4, His), 7.52 (1H, d, $J=7.8 \mathrm{~Hz}, \mathrm{H}-2$, Try), 7.39 (1H, d, $J=7.9 \mathrm{~Hz}, \mathrm{H}-2$, His), 7.15-7.08 (3H, m, H-5-7,

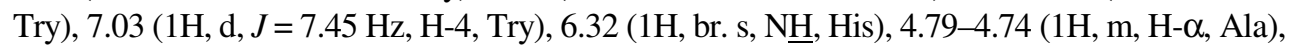


4.24-4.18 (1H, m, H- $\alpha$, Try), 4.17-4.12 (1H, m, H- $\alpha$, His), 3.79 (2H, d, J = 5.2 Hz, H- $\alpha$, Gly), 3.54 ( $\left.3 \mathrm{H}, \mathrm{s}, \mathrm{OC}_{3}\right), 3.21(2 \mathrm{H}, \mathrm{d}, J=5.8 \mathrm{~Hz}, \mathrm{H}-\beta$, Try), 2.83 ( $2 \mathrm{H}, \mathrm{d}, J=5.85 \mathrm{~Hz}, \mathrm{H}-\beta, \mathrm{His}), 1.25$ (3H, d, $J=5.95 \mathrm{~Hz}, \mathrm{H}-\beta$, Ala); MASS (m/z \%RI): 951(2.8), 950( $\left.\mathrm{M}^{+}, 4.3\right), 935(15.8), 919(29.6)$, 891(14.9), 733(54.6), 705(22.3), 676(100), 648(19.4), 539(72.8), 511(21.4), 467(55.2), 439(23.7), 328(10.7), 203(9.5), 159(15.8), 130(13.3), 116(6.9), 113(11.6), 112(15.9), 110(19.5), 86(16.6), 85(8.7), 81(4.8), 67(9.2), 59(17.7), 46(7.9), 44(15.5), 31(11.8), 30(8.7), 15(1.9); Anal. calcd. for $\mathrm{C}_{33} \mathrm{H}_{32} \mathrm{I}_{2} \mathrm{~N}_{10} \mathrm{O}_{8}$ (950): C, 41.70; H, 3.39; N, 14.74. Found: C, 41.69; H, 3.36; N, $14.75 \%$.

Preparation of 3,5-diiodo-4-(5-nitro-1H-2-imidazolyl)benzoyl amino acids and peptides (12-15)

To a solution of the amino acid/peptide methyl esters $(0.01 \mathrm{~mol})$ in THF: $\mathrm{H}_{2} \mathrm{O}(1: 1,36 \mathrm{~mL})$, $\mathrm{LiOH}(0.36 \mathrm{gm})$ was added at $0{ }^{\circ} \mathrm{C}$. The mixture was stirred at room temperature for $1 \mathrm{~h}$ and then acidified to $\mathrm{pH} 3.5$ with $1 \mathrm{~N} \mathrm{H}_{2} \mathrm{SO}_{4}$. The aqueous layer was extracted with $\mathrm{Et}_{2} \mathrm{O}(3 \times 25$ $\mathrm{mL}$ ). The combined organic extracts were dried over anhydrous $\mathrm{Na}_{2} \mathrm{SO}_{4}$ and concentrated under reduced pressure. The crude products were crystallized from methanol and ether to get hydrolyzed peptide derivatives.

\section{3,5-diiodo-4-(5-nitro-1H-2-imidazolyl)benzoyl tyrosine (12)}

White crystals; Yield 89\%; m.p. 77-78 ${ }^{\circ} \mathrm{C} ;[\alpha]_{\mathrm{D}}-103.6^{\circ}$ (c 0.5, $\left.\mathrm{MeOH}\right) ; \mathrm{R}_{f}-0.74\left(\mathrm{CHCl}_{3}: \mathrm{MeOH}\right.$ / 7:3); IR (KBr) $\left(\mathrm{cm}^{-1}\right)$ : $3486(\mathrm{~N}-\mathrm{H}$ str, ring), $3355(\mathrm{O}-\mathrm{H}$ str, $\mathrm{Ar}-\mathrm{OH}), 3298-2510(\mathrm{O}-\mathrm{H} \mathrm{str}$, $\mathrm{COOH}$ ), 3125 (N-H str, amide), 3075, 3056, 3053 (Ar-H str), 2928, 2853 (C-H str, $\mathrm{CH}_{2}$ ), 1711 (C=O str, $\mathrm{COOH}), 1644$ (C=O str, amide), 1587-1579, 1423-1419 (skeletal bands), 1543, 1348 $\left(\mathrm{NO}_{2}\right.$ str), 1533 (N-H def, amide), 1405 (O-H def, $\left.\mathrm{COOH}\right), 1227$ (C-O str, phenolic), 869, 829-

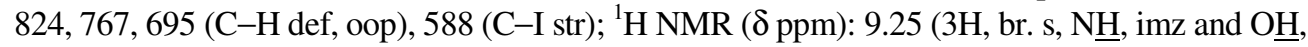
Tyr and $\mathrm{COO} \underline{\mathrm{H}}), 8.65(1 \mathrm{H}$, br. s, $\underline{\mathrm{H}}), 8.39$ (2H, s, H-2 and H-6, iba), 7.83 (1H, s, H- $\delta, \mathrm{imz})$, 7.19 (2H, dd, $J=8.6,5.25 \mathrm{~Hz}, \mathrm{H}-\mathrm{o}, \mathrm{Tyr}), 6.77$ (2H, dd, $J=8.65,4.85 \mathrm{~Hz}, \mathrm{H}-\mathrm{m}, \mathrm{Tyr}), 4.90-4.86$ $(1 \mathrm{H}, \mathrm{m}, \mathrm{H}-\alpha, \mathrm{Tyr}), 2.93(2 \mathrm{H}, \mathrm{d}, J=5.5 \mathrm{~Hz}, \mathrm{H}-\beta, \mathrm{Tyr}) ;{ }^{13} \mathrm{C} \mathrm{NMR}(\delta \mathrm{ppm}): 173.2(\mathrm{C}=\mathrm{O}, \mathrm{Tyr})$,

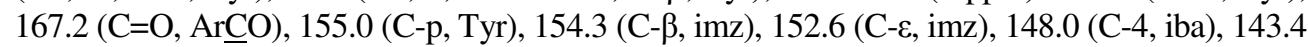
(2C, C-2 and C-6, iba), 140.3 (C-1, iba), 139.4 (2C, C-m, Tyr), 137.2 (C- $\delta, i m z), 128.6$ (2C, C-o, Tyr), 125.3 (C- $\gamma$, Tyr), 109.1 (2C, C-3 and C-5, iba), 53.0 (C- $\alpha$, Tyr), 37.2 (C- $\beta$, Tyr); Anal. calcd. for $\mathrm{C}_{19} \mathrm{H}_{14} \mathrm{I}_{2} \mathrm{~N}_{4} \mathrm{O}_{6}(648)$ : C, 35.21; H, 2.18; N, 8.64. Found: C, 35.24; H, 2.20; N, 8.65\%.

\section{3,5-Diiodo-4-(5-nitro-1H-2-imidazolyl)benzoyl tryptophanyl-histidine (13)}

White solid; Yield 80\%; m.p. $175^{\circ} \mathrm{C} ;[\alpha]_{\mathrm{D}}+12.6^{\circ}$ (c 0.5, $\left.\mathrm{MeOH}\right) ; \mathrm{R}_{f}-0.72\left(\mathrm{CHCl}_{3}: \mathrm{MeOH} / 7: 3\right)$; IR (KBr) $\left(\mathrm{cm}^{-1}\right)$ : 3488, 3485, 3483 (N-H str, rings), 3306-2513 (O-H str, COOH), 3126, 3118 (N-H str, amide), 3077-3073, 3056 (Ar-H str), 2927-2924, 2852, 2849 (C-H str, $\mathrm{CH}_{2}$ ), 1709 $(\mathrm{C}=\mathrm{O}$ str, $\mathrm{COOH}), 1643,1640$ (C=O str, amide), 1585-1576, 1429-1417 (skeletal bands), 1540, $1342\left(\mathrm{NO}_{2}\right.$ str), 1537-1533 (N-H def, amide), 1466 (C-H def, $\left.\mathrm{CH}_{2}\right), 1409$ (O-H def, $\left.\mathrm{COOH}\right)$, 879-874, 830-825, 696 (C-H def, oop), 588 (C-I str); ${ }^{1} \mathrm{H}$ NMR ( $\delta$ ppm): 9.95 (4H, br s, N $\underline{H}, \mathrm{imz}$, His, Try and $\mathrm{OH}, \mathrm{COOH}), 9.34(1 \mathrm{H}$, br. s, $\mathrm{N} \underline{\mathrm{H}}, \mathrm{Try}), 8.50(2 \mathrm{H}, \mathrm{s}, \mathrm{H}-2$ and $\mathrm{H}-6, \mathrm{iba}), 8.05(1 \mathrm{H}, \mathrm{d}$, $J=7.9 \mathrm{~Hz}, \mathrm{H}-2, \mathrm{His}), 7.85(1 \mathrm{H}, \mathrm{s}, \mathrm{H}-\delta$, imz), $7.65(1 \mathrm{H}, \mathrm{s}, \mathrm{H}-4, \mathrm{His}), 7.26(1 \mathrm{H}, \mathrm{d}, J=7.8 \mathrm{~Hz}, \mathrm{H}-2$, Try), $7.23(1 \mathrm{H}$, br. s, N $\underline{H}$, His), $7.20(1 \mathrm{H}, \mathrm{d}, J=7.45 \mathrm{~Hz}, \mathrm{H}-4$, Try), 7.14-7.08 (3H, m, H-5-7, Try), 5.89-5.84 (1H, m, H- $\alpha$, Try), 4.38-4.33 (1H, m, H- $\alpha$, His), 3.22 (2H, d, J = 5.7 Hz, H- $\beta$, Try), 3.09 (2H, d, $J=5.85 \mathrm{~Hz}, \mathrm{H}-\beta, \mathrm{His}) ;{ }^{13} \mathrm{C}$ NMR $(\delta \mathrm{ppm}): 179.4(\mathrm{C}=\mathrm{O}, \mathrm{His}), 172.0(\mathrm{C}=\mathrm{O}$, Try), 170.4 (C=O, ArCO $), 154.6$ (C- $\beta$, imz), 152.9 (C- $\varepsilon$, imz), 149.0 (C-2, His), 148.2 (C-4, iba), 143.0 (2C, C-2 and C-6, iba), 140.1 (C-1, iba), 137.4 (C- $\delta$, imz), 136.0 (C-2', Try), 133.3 (C-4, His), 129.0 (C-3', Try), 124.4 (C-2, Try), 123.9, 122.7 (2C, C-5 and C-6, Try), 119.3 (C-4, Try), 
116.2 (C-5, His), 112.5 (C-3, Try), 111.0 (C-7, Try), 108.4 (2C, C-3 and C-5, iba), 67.6 (C- $\alpha$, Try), 58.3 (C- $\alpha$, His), 32.4 (C- $\beta$, His), 24.0 (C- $\beta$, Try); Anal. calcd. for $\mathrm{C}_{27} \mathrm{H}_{22} \mathrm{I}_{2} \mathrm{~N}_{8} \mathrm{O}_{6}$ (808): C, 40.12; H, 2.74; N, 13.86. Found: C, 40.10; H, 2.75; N, $13.85 \%$.

\section{3,5-Diiodo-4-(5-nitro-1H-2-imidazolyl)benzoyl prolyl-prolyl-proline (14)}

Semisolid mass; Yield 85\%; $[\alpha]_{\mathrm{D}}+47.1^{\circ}(\mathrm{c} 0.5, \mathrm{MeOH}) ; \mathrm{R}_{f}-0.75\left(\mathrm{CHCl}_{3}: \mathrm{MeOH} / 7: 3\right)$; IR $\left(\mathrm{CDCl}_{3}\right)\left(\mathrm{cm}^{-1}\right): 3486(\mathrm{~N}-\mathrm{H}$ str, ring), 3293-2515 (O-H str, $\mathrm{COOH}), 3079,3053$ ( $\mathrm{Ar}-\mathrm{H}$ str), 2997-2987 (C-H str, $\mathrm{CH}_{2}$, Pro), 1705 (C=O str, $\mathrm{COOH}$ ), 1663-1656 (C=O str, amide), 1587, 1574, 1427, 1418 (skeletal bands), 1547, 1346 ( $\mathrm{NO}_{2}$ str), 1406 (O-H def, $\left.\mathrm{COOH}\right), 878,829-825,696$ (C-H def, oop), 588 (C-I str); ${ }^{13} \mathrm{C}$ NMR ( $\delta$ ppm): 178.4 (C=O, Pro-3), 171.7 (C=O, Pro-1), 169.9 $(\mathrm{C}=\mathrm{O}, \operatorname{Ar} \underline{\mathrm{CO}}), 167.6$ (C=O, Pro-2), 154.9 (C- $\beta, \mathrm{imz}), 152.8$ (C- $\varepsilon, \mathrm{imz}), 148.0(\mathrm{C}-4, \mathrm{iba}), 143.8$ (2C, C-2 and C-6, iba), 140.2 (C-1, iba), 137.5 (C- $\delta$, imz), 108.9 (2C, C-3 and C-5, iba), 64.1, 59.5, 57.9 (3C, C- $\alpha$, Pro-1-3), 47.7, 46.4, 44.2 (3C, C- $\delta$, Pro-1-3), 31.2, 29.7, 29.0 (3C, C- $\beta$, Pro-1-3), 27.5, 25.0, 23.8 (3C, C- $\gamma$, Pro-1-3); MASS (m/z \%RI): 777(2.7), 776( $\left.\mathrm{M}^{+}, 4.2\right)$, 759(35.3), 731(19.8), 662(63.5), 634(17.8), 565(100), 537(20.4), 467(60.8), 439(25.9), 328(15.6), 203(13.5), 126(7.3), 113(11.4), 112(17.4), 86(19.9), 85(6.7), 46(7.8), 45(10.6), 42(15.9), 15(3.2); Anal. calcd. for $\mathrm{C}_{25} \mathrm{H}_{26} \mathrm{I}_{2} \mathrm{~N}_{6} \mathrm{O}_{7}$ (776): C, 38.68; H, 3.38; N, 10.83. Found: C, 38.69; H, 3.35; N, $10.85 \%$.

\section{3,5-Diiodo-4-(5-nitro-1H-2-imidazolyl)benzoyl alanyl-histidinyl-glycyl-tryptophan (15)}

Semisolid mass; Yield 88\%; $[\alpha]_{\mathrm{D}}-81.3^{\circ}(\mathrm{c} 0.5, \mathrm{MeOH}) ; \mathrm{R}_{f}-0.66\left(\mathrm{CHCl}_{3}: \mathrm{MeOH} / 7: 3\right)$; IR $\left(\mathrm{CDCl}_{3}\right)\left(\mathrm{cm}^{-1}\right): 3488,3485(\mathrm{~N}-\mathrm{H}$ str, rings), 3295-2502 (O-H str, $\mathrm{COOH}), 3126-3119(\mathrm{~N}-\mathrm{H}$ str, amide), 3079-3076, 3056 ( $\mathrm{Ar}-\mathrm{H}$ str), 2967, 2870 (C-H str, $\mathrm{CH}_{3}$ ), 2928-2924, 2850, 2846 (C-H str, $\left.\mathrm{CH}_{2}\right), 1708$ (C=O str, $\left.\mathrm{COOH}\right), 1642-1638$ (C=O str, amide), 1587-1579, 1427-1419 (skeletal bands), 1540, 1347 ( $\mathrm{NO}_{2}$ str), 1536-1531 (N-H def, amide), 1465 (C-H def, $\left.\mathrm{CH}_{2}\right), 1408(\mathrm{O}-\mathrm{H}$ def, $\mathrm{COOH}), 877-872,833-827,695$ (C-H def, oop), 586 (C-I str); ${ }^{1} \mathrm{H}$ NMR $(\delta \mathrm{ppm}): 9.98(1 \mathrm{H}$, br. s, $\underline{\mathrm{H}}$, His), 9.92 (4H, br. s, NH, imz, His, Try and $\mathrm{OH}, \mathrm{COO} \underline{\mathrm{H}}), 8.74(1 \mathrm{H}$, br. s, $\underline{\mathrm{H}}, \mathrm{Ala})$, 8.48 (2H, s, H-2 and H-6, iba), 8.23 (1H, br. s, NH, Gly), $7.86(1 \mathrm{H}, \mathrm{s}, \mathrm{H}-\delta$, imz), 7.67 (1H, s, H-4, His), $7.56(1 \mathrm{H}, \mathrm{d}, J=7.75 \mathrm{~Hz}, \mathrm{H}-2$, Try $), 7.38(1 \mathrm{H}, \mathrm{d}, J=7.85 \mathrm{~Hz}, \mathrm{H}-2, \mathrm{His}), 7.22-7.18(2 \mathrm{H}, \mathrm{m}$,

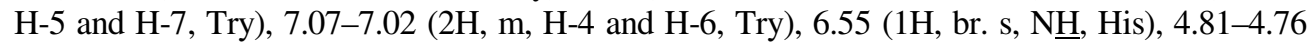
(1H, m, H- $\alpha$, Ala), 4.48-4.44 (1H, m, H- $\alpha$, Try), 4.16-4.11 (1H, m, H- $\alpha$, His), 3.82 (2H, d, $J=$ $5.15 \mathrm{~Hz}, \mathrm{H}-\alpha$, Gly), 3.23 (2H, d, $J=5.75 \mathrm{~Hz}, \mathrm{H}-\beta$, Try), 2.87 (2H, d, $J=5.9 \mathrm{~Hz}, \mathrm{H}-\beta$, His), 1.27 (3H, d, $J=5.9 \mathrm{~Hz}, \mathrm{H}-\beta$, Ala); MASS (m/z \%RI): 937(1.7), 936( $\left.\mathrm{M}^{+}, 3.5\right), 919(18.7), 891(33.6)$, 733(44.9), 705(20.8), 676(100), 648(24.4), 539(69.2), 511(19.2), 467(58.8), 439(21.2), 328(14.4), 203(11.7), 159(12.9), 130(16.8), 116(9.2), 113(15.6), 112(13.5), 110(15.9), 86(18.8), 85(7.6), 81(5.9), 67(7.8), 46(9.2), 45(11.9), 44(13.7), 30(7.8), 15(2.2); Anal. calcd. for $\mathrm{C}_{32} \mathrm{H}_{30} \mathrm{I}_{2} \mathrm{~N}_{10} \mathrm{O}_{8}$ (936): C, 41.04; H, 3.23; N, 14.96. Found: C, 41.02; H, 3.25; N, $15.00 \%$.

\section{Results and Discussion}

All peptide derivatives (4-15) were synthesized in good yields using DIPC/DCC as coupling agents and NMM/TEA as bases. Presence of bands at 3302-2497, 1698, 1545, 1348, 590 $\mathrm{cm}^{-1}$ in IR spectra of compound (3) clearly indicated presence of functional groups like $\mathrm{COOH},-\mathrm{NO}_{2}$ and $-\mathrm{I}$ but no free $-\mathrm{NH}_{2}$ group which was present in the starting material (1). Furthermore, IR spectra of peptide derivatives (4-15) showed amide I and amide II bands at $1663-1638 \mathrm{~cm}^{-1}$ and $1537-1529 \mathrm{~cm}^{-1}$ indicating formation of peptide bonds and accomplishment of coupling reaction. This fact was further confirmed by appearance of broad singlets at 9.98-6.32 ppm (for imino proton of CO-N $\underline{H}$ moiety) in ${ }^{1} \mathrm{H}$ NMR spectra and singlets at 179.4-165.1 ppm (for carbonyl carbon of $\underline{\mathrm{CO}}-\mathrm{NH}$ moiety) in ${ }^{13} \mathrm{C} \mathrm{NMR}$ 
spectra of compounds (4-15). Moreover, presence of nitro and iodo groups in peptide analogs of compound (3) was indicated by appearance of medium bands at $1549-1540 \mathrm{~cm}^{-1}$ and $1349-1341$ $\mathrm{cm}^{-1}$ (asymmetric and symmetric $\mathrm{NO}_{2}$ stretching), and at $589-585 \mathrm{~cm}^{-1}$ (C-I str) in IR spectra. Mass spectra of peptide ester derivatives showed molecular ion peaks along with isotopic peaks at $\mathrm{m} / \mathrm{z}$ values, consistent with their respective molecular formulas. All peptide ester derivatives showed easily distinguishable $\mathrm{R}-\mathrm{C} \equiv \mathrm{O}^{+}$ion peaks at $\mathrm{M}-31$ along with characteristic fragmentation pattern after and before the carbonyl moiety in their respective structures. Furthermore, $\left[\mathrm{CH}_{3} \mathrm{O}^{+}\right]$and $\left[\mathrm{CH}_{3} \mathrm{OCO}^{+}\right]$fragment ion peaks appeared at $\mathrm{m} / z$ values 31 and 59 in mass spectra of synthesized peptide ester derivatives. Structures of hydrolyzed derivatives (12-15) were confirmed by appearance of strong bands at $1711-1705 \mathrm{~cm}^{-1}(\mathrm{C}=\mathrm{O}$ str, $\underline{\mathrm{COOH}})$ in IR spectra and broad singlets at 9.95-9.25 ppm (for hydroxyl proton of $\mathrm{COO} \underline{\mathrm{H}}$ ) in ${ }^{1} \mathrm{H}$ NMR spectra. This fact was further supported by disappearance of medium to strong bands at $1751-1749 \mathrm{~cm}^{-1}$ $\left(\mathrm{C}=\mathrm{O}\right.$ str, ester) and $1273-1270 \mathrm{~cm}^{-1}(\mathrm{C}-\mathrm{O}$ str, ester $)$ in IR spectra and singlets at 54.4-51.4 ppm (for carbonyl carbon of $\mathrm{OCH}_{3}$ ) in ${ }^{13} \mathrm{C}$ NMR spectra of compounds (12-15). All hydrolyzed peptide derivatives showed peaks at $\mathrm{M}-17$ and $\mathrm{M}-45$ in their mass spectra by loss of $\mathrm{OH}$ and $\mathrm{COOH}$ respectively. Moreover, $\left[\mathrm{COOH}^{+}\right]$fragment ion peak appeared at $\mathrm{m} / z$ value 45 in mass spectra of compounds $(14,15)$ along with characteristic fragmentation pattern after and before the carbonyl moiety. The newly synthesized compounds were also analyzed for $\mathrm{C}, \mathrm{H}$ and $\mathrm{N}$ content and the results revealed the variation by a factor of \pm 0.03 from calculated values.

\section{Biological activity}

All the synthesized compounds were subjected to anthelmintic activity studies ${ }^{30}$ against three different species of earthworms M. konkanensis, P. corethruses and E. eugeniea at $2 \mathrm{mg} \mathrm{mL}^{-1}$ concentration. Tween $80(0.5 \%)$ in distilled water was used as control whereas albendazole and mebendazole were used as a reference compounds. The paralyzing and death times were noted and their mean was calculated for triplicate sets. The death time was ascertained by placing the earthworms in warm water $\left(50^{\circ} \mathrm{C}\right)$ which stimulated the movement, if the worm was alive. The results of anthelmintic studies are tabulated in Table 1.

Table 1. Anthelmintic activity of compounds (4-15).

\begin{tabular}{|c|c|c|c|c|c|c|}
\hline \multirow[b]{3}{*}{ Compound } & \multicolumn{6}{|c|}{ Earthworm species } \\
\hline & \multicolumn{2}{|c|}{ M. konkanensis } & \multicolumn{2}{|c|}{ P. corethruses } & \multicolumn{2}{|c|}{ E. eugeniea } \\
\hline & $\begin{array}{l}\text { Mean } \\
\text { paralyzing } \\
\text { time, } \min ^{\dagger}\end{array}$ & $\begin{array}{l}\text { Mean } \\
\text { death } \\
\text { time, } \min ^{\dagger}\end{array}$ & $\begin{array}{l}\text { Mean } \\
\text { paralyzing } \\
\text { time, min }\end{array}$ & $\begin{array}{l}\text { Mean } \\
\text { death } \\
\text { time, min }\end{array}$ & $\begin{array}{l}\text { Mean } \\
\text { paralyzing } \\
\text { time, min }\end{array}$ & $\begin{array}{l}\text { Mean } \\
\text { death } \\
\text { time, min }\end{array}$ \\
\hline 4 & $40.58 \pm 0.12$ & $51.58 \pm 0.59$ & $44.57 \pm 0.26$ & $56.26 \pm 0.42$ & $42.25 \pm 0.23$ & $54.34 \pm 0.62$ \\
\hline 5 & $17.56 \pm 0.28$ & $26.56 \pm 0.15$ & $21.65 \pm 0.44$ & $33.22 \pm 0.17$ & $16.67 \pm 0.82$ & $26.21 \pm 0.12$ \\
\hline 6 & $14.34 \pm 0.19$ & $24.40 \pm 0.84$ & $18.17 \pm 0.88$ & $30.40 \pm 0.13$ & $16.73 \pm 0.49$ & $25.54 \pm 0.93$ \\
\hline 7 & $33.68 \pm 0.12$ & $45.59 \pm 0.72$ & $39.55 \pm 0.23$ & $52.18 \pm 0.17$ & $37.49 \pm 0.32$ & $49.28 \pm 0.44$ \\
\hline 8 & $31.22 \pm 0.21$ & $42.48 \pm 0.16$ & $35.35 \pm 0.65$ & $47.24 \pm 0.54$ & $33.45 \pm 0.58$ & $46.34 \pm 0.62$ \\
\hline 9 & $10.25 \pm 0.44$ & $18.52 \pm 0.43$ & $12.22 \pm 0.24$ & $23.50 \pm 0.42$ & $11.59 \pm 0.41$ & $22.10 \pm 0.60$ \\
\hline 10 & $28.15 \pm 0.52$ & $41.29 \pm 0.76$ & $32.04 \pm 0.12$ & $42.96 \pm 0.54$ & $29.38 \pm 0.69$ & $38.12 \pm 0.71$ \\
\hline 11 & $15.29 \pm 0.19$ & $24.27 \pm 0.92$ & $19.61 \pm 0.21$ & $32.39 \pm 0.20$ & $16.80 \pm 0.15$ & $27.24 \pm 0.55$ \\
\hline 12 & $16.54 \pm 0.81$ & $24.18 \pm 0.28$ & $20.47 \pm 0.47$ & $31.02 \pm 0.18$ & $15.44 \pm 0.89$ & $24.58 \pm 0.38$ \\
\hline 13 & $08.28 \pm 0.17$ & $17.46 \pm 0.28$ & $10.14 \pm 0.23$ & $21.82 \pm 0.51$ & $09.34 \pm 0.56$ & $20.30 \pm 0.19$ \\
\hline 14 & $26.04 \pm 0.53$ & $38.28 \pm 0.71$ & $30.10 \pm 0.38$ & $38.29 \pm 0.30$ & $27.50 \pm 0.11$ & $36.02 \pm 0.66$ \\
\hline 15 & $14.23 \pm 0.56$ & $23.23 \pm 0.38$ & $18.60 \pm 0.12$ & $30.32 \pm 0.37$ & $14.22 \pm 0.74$ & $25.45 \pm 0.48$ \\
\hline Control $^{+}$ & - & - & - & - & - & - \\
\hline Albendazole & $11.25 \pm 0.41$ & $19.45 \pm 0.26$ & $13.20 \pm 0.22$ & $24.23 \pm 0.46$ & $12.33 \pm 0.50$ & $23.35 \pm 0.28$ \\
\hline Mebendazole & $13.85 \pm 0.64$ & $22.85 \pm 0.53$ & $17.82 \pm 0.43$ & $29.60 \pm 0.22$ & $13.54 \pm 0.45$ & $24.05 \pm 0.62$ \\
\hline
\end{tabular}


Almost, all the newly synthesized compounds were found to exhibit moderate to good anthelmintic activity against all the three earthworm species. Comparison of anthelmintic activity data revealed that hydrolyzed peptide derivatives (12-15) are slightly more active than their corresponding ester derivatives (5, 9-11). Compound (9) and its hydrolyzed derivative (13) were found to exhibit higher bioactivity against all three earthworm species, in comparison to standard drugs-albendazole and mebendazole whereas compound (4) displayed least anthelmintic activity. Moreover, compounds $(6,9,11,13$ and 15) bearing tryptophan and histidine residues in their amino acid chains were found to possess good activity against all earthworms species whereas compounds $(7,8,10$ and 14$)$ showed only moderate level of activity.

\section{Conclusions}

Present investigation describes successful synthesis of title compounds via coupling reaction in good yields. For peptide coupling, method employing DIPC/NMM proved to be better than DCC/TEA method utilizing DMF as solvent, providing 6-10\% extra yields. Diisopropyl carbodiimide proved to be a good coupling agent both economically and yieldwise. Greater anthelmintic activity was found in derivatives with tryptophan and histidine constituents in their amino acid chain. Hydrolyzed derivatives exhibited more anthelmintic activity in comparison to their corresponding methyl ester analogs. Among tested compounds (9,13 and 15) possessed better anthelmintic activity as compared to standard drugs. On passing toxicity tests, these compounds may prove good candidates for clinical studies and may be potential anthelmintic agents of future.

\section{Acknowledgement}

Authors are thankful to U.S.I.C., DU, Delhi (India) for spectral and elemental analysis. Also great thanks to C.P.C.R.I., Kasaragod, Kerala (India) for providing earthworms for testing anthelmintic activity.

\section{References}

1. Aridoss G, Balasubramanian S, Parthiban P and Kabilan S, Eur J Med Chem., 2006, 41, 268.

2. Nagarapu L, Satyender A, Rajashaker B, Srinivas K, Rani P R, Radhika K and Subhashini G, Bioorg Med Chem Lett., 2008, 18, 1167.

3. Navidpour L, Shadnia H, Shafaroodi H, Amini M, Dehpour A R and Shafiee A, Bioorg Med Chem., 2007, 15, 1976.

4. Ucucu U, Karaburun N G and Isikdag I, Il Farmaco, 2001, 56, 285.

5. Gupta P, Hameed S and Jain R, Eur J Med Chem., 2004, 39, 805.

6. Arrowsmith J, Jennings S A, Clark A S and Stevens M F G, J Med Chem., 2002, 45, 5458.

7. Wang Y, Inguaggiato G, Jasamai M, Shah M, Hughes D, Slater M and Simons C Bioorg Med Chem., 1999, 7, 481.

8. Miyachi H, Kiyota H and Segawa M, Bioorg Med Chem Lett., 1998, 8, 2163.

9. Angibaud P, Bourdrez X, Devine A, End D W, Freyne E, Ligny Y, Muller P, Mannens G, Pilatte I, Poncelet V, Skrzat S, Smets G, Dun J V, Remoortere P V, Venet M and Wouters W, Bioorg Med Chem Lett., 2003, 13, 1543.

10. Yamada M, Yura T, Morimoto M, Harada T, Yamada K, Honma Y, Kinoshita M and Sugiura M, J Med Chem., 1996, 39, 596.

11. Dobler M R, Tetrahedron Lett., 2003, 44, 7115.

12. Kiselyou A S, Tetrahedron Lett., 2006, 47, 2941.

13. Balalaie S, Hashemi M M and Akhbari M, Tetrahedron Lett., 2003, 44, 1709.

14. Dahiya R, Ethiop Pharm J., 2008, 26, 17.

15. Dahiya R, Sci Pharm., 2008, 76, 217. 
16. Dahiya R, Pathak D and Kaur R, J Indian Chem Soc., 2008, 85, 754.

17. Dahiya R, Kumar A and Yadav R, Molecules, 2008, 13, 958.

18. Dahiya R and Kaur R, Int J Biol Chem Sci., 2008, 2, 1.

19. Dahiya R and Pathak D, Eur J Med Chem., 2007, 42, 772.

20. Dahiya R and Kaur R, Biosci Biotech Res Asia., 2007, 4, 561.

21. Dahiya R and Kaur R, Aust J Basic Appl Sci., 2007, 1, 525.

22. Dahiya R and Pathak D, Indian J Heterocycl Chem., 2006, 16, 53-56.

23. Dahiya R, Pathak D and Bhatt S, J Saudi Chem Soc., 2006, 10, 165.

24. Dahiya R and Pathak D, J Nepal Pharm Assoc., 2006, XXIV, 17.

25. Dahiya R, Pathak D and Bhatt S, Bull Chem Soc Ethiop., 2006, 20, 235.

26. Dahiya R and Pathak D, Orient J Chem., 2006, 22, 123.

27. Vogel A, Textbook of Practical Organic Chemistry; Pearson Education:Singapore, 2004, 909.

28. Mann F G and Saunders B C, Practical Organic Chemistry; Orient Longman:New Delhi, 2001, 393.

29. Bodanzsky M and Bodanzsky A, The Practice of Peptide Synthesis; SpringerVerlag:New York, 1984, 68.

30. Garg L C and Atal C K, Indian J Pharm. Sci., 1963, 59, 240. 


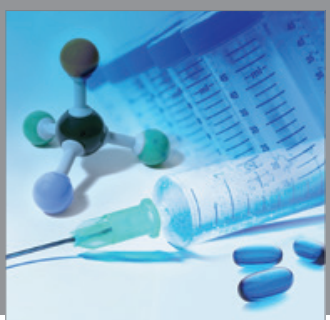

International Journal of

Medicinal Chemistry

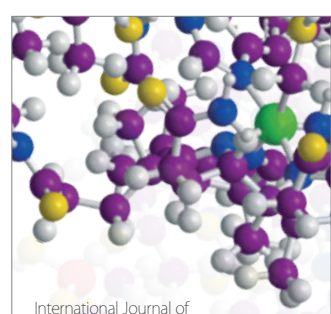

Carbohydrate Chemistry

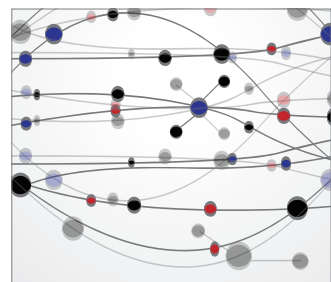

The Scientific World Journal
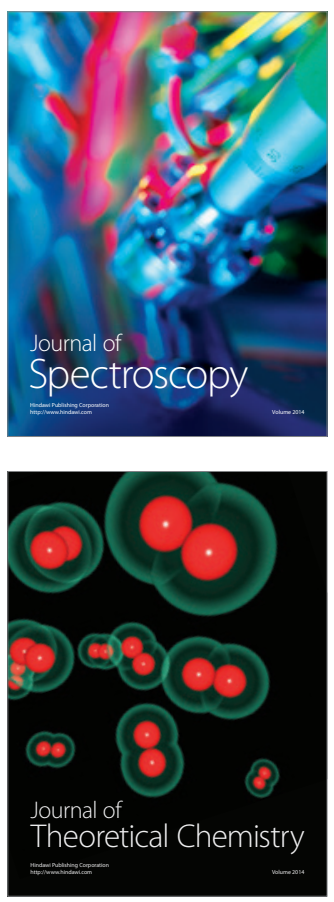
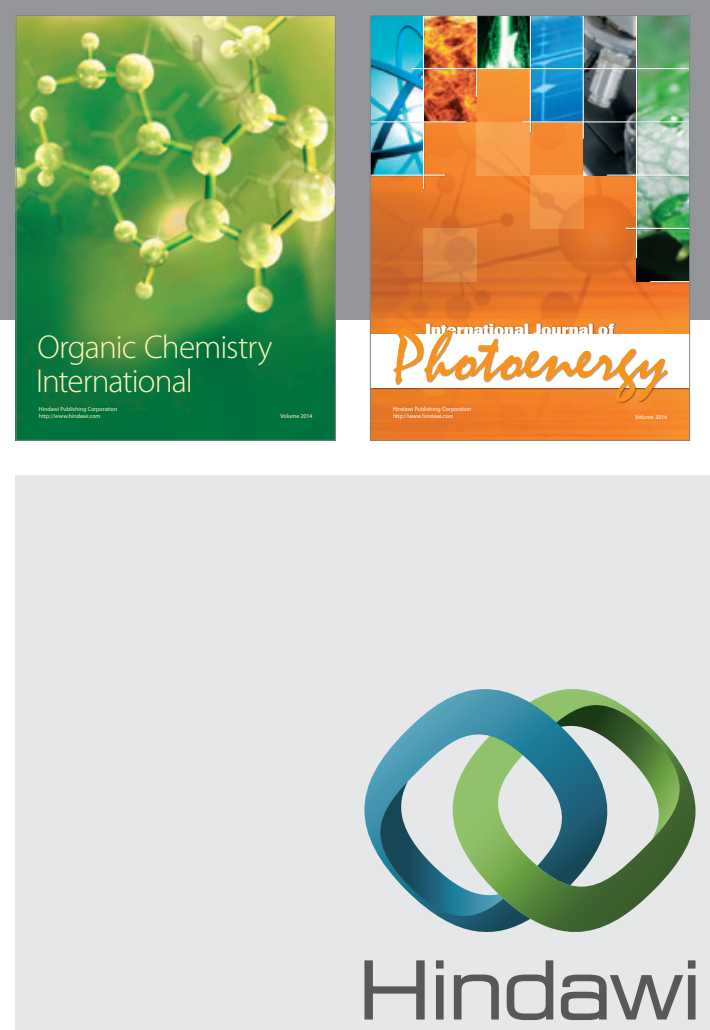

Submit your manuscripts at

http://www.hindawi.com
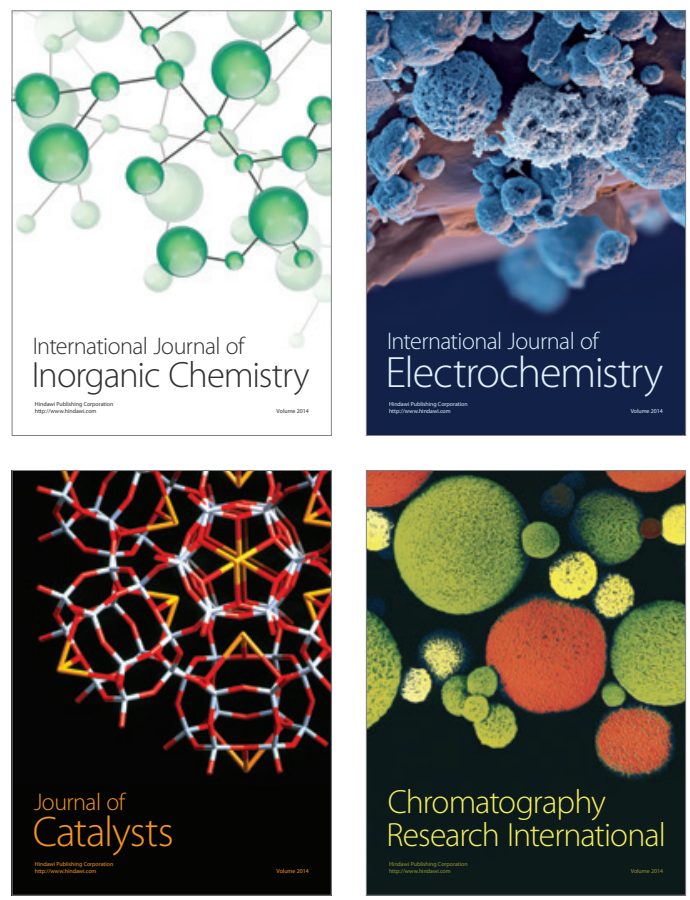
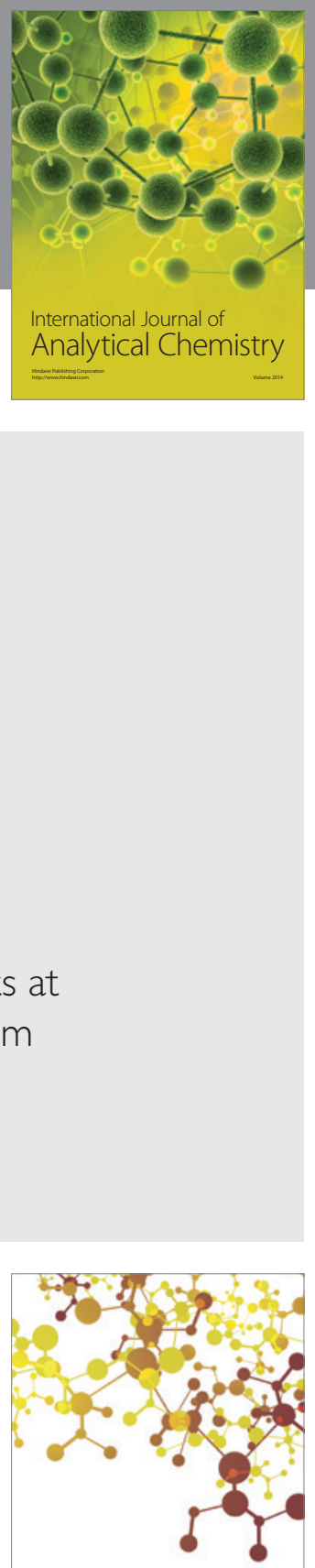

Journal of

Applied Chemistry
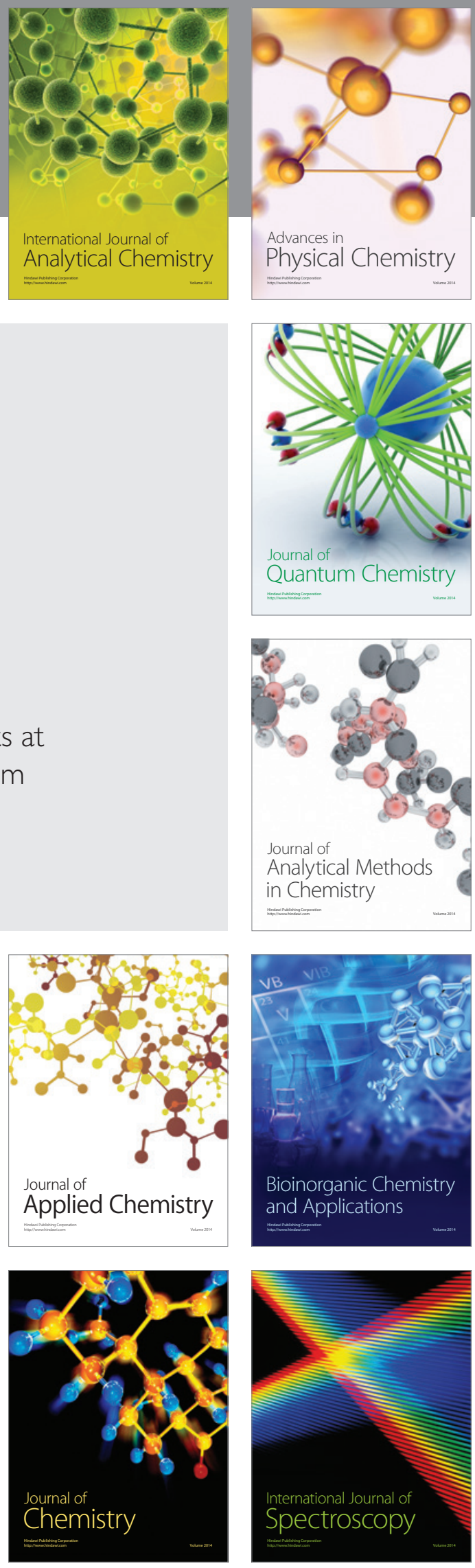